\title{
Improving Clinical fMRI: Better Paradigms or Higher Field Strength?
}

$\mathbf{T}$ he clinical significance of functional MR imaging is steadily increasing. At the latest annual meeting of the Organization for Human Brain Mapping (www.humanbrainmapping.org) in Seattle this year, the largest group of contributions concerned the category "Disorders of the Nervous System." In addition, specialized clinical associations have been founded in recent years (eg, www.asfnr.org and www.oegfmrt.org); and at clinical centers with dedicated expertise, the demand for clinical fMRI reports is steadily growing (exact quantifications are difficult due to the absence of international statistics). The most important tasks for clinical fMRI are the generation of noninvasive biomarkers for the classification and prognosis of patients with brain disease and the presurgical localization of essential motor, language, or memory areas. Research concerning possible improvements of current clinical fMRI techniques is highly topical. Two distinctive features render this research difficult: First, the techniques need to generate reliable data in individual patients. Second, the techniques need to work with atypical morphology and functionality (ie, pathologic brains).

The consequence of these features is that clinical fMRI has to struggle with several issues that are less problematic in research with healthy subjects. Among these are patient compliance, performance monitoring, recognition of neuroplastic changes, and evaluation of compensatory activations that may result in a changed lateralization index. The latter is particularly problematic because a clinically valid interpretation of lateralized activation in pathologic brains is not trivial. Recent neuroimaging research indicates that there are 2 types of contralateral activation increase after ipsilateral brain damage. If much of the functional capacity is lost in the damaged hemisphere, contralateral activations may be essential and inhibition of this activity may be deleterious. In contrast, if sufficient functional capacity is left in the damaged hemisphere, contralateral activations may represent maladaptations and their inhibition improves patient performance. $^{1,2}$ Differentiating these 2 types of compensatory activation is not straightforward. Important issues in this context are the patient's clinical performance and the time elapsed since brain damage. Particular caution is required when results indicate an atypical functional lateralization. Evidently this situation requires a comprehensive evaluation of the individual case but also highly reliable functional data.

Considerable research within the past decade has focused on improving the reliability of clinical fMRI data. Much of it concerns the important issue of how to optimize experimental paradigms. Especially for localization of essential language areas, many different paradigms have already been suggested. However, only a few comprise the most important issues of performance monitoring and comprehensive testing of the various subcomponents of language processing. A nice example is included in the present issue of the American Journal of Neuroradiology in the article by Zacà et al. ${ }^{3}$ This study-like most paradigm research - is based on healthy subjects, and unfortunately, much less data are available from pathologic brains or typical clinical fMRI populations. Even worse is the situation with research on the issue of how to optimize signal strength when a well-functioning paradigm is already in place. Given that functional signals within pathologic tissue may be rather weak and given the clinical implications of defining a brain area as functionally "dead" as opposed to functionally "compromised," the issue of optimizing signal strength is highly important. This is particularly true for the clinical demands that require a diagnosis in an individual without the possibility of averaging over a group. Due to the approximately linear increase of the signal-to-noise ratio from 1.5T to 7T, increasing the magnetic field strength of MR imaging systems is one of the key issues in this regard. If one considers MR imaging history, approximately 1 decade ago, $1.5 \mathrm{~T}$ was the maximum field strength used for clinical applications. Currently $3 \mathrm{~T}$ clinical systems are widely available and quasistandard for fMRI investigations. The cutting edge technology, however, is 7T ultra-highfield systems, with approximately 60 installations worldwide.

Besides an increased signal-to-noise ratio, theoretic expectations also include benefits for the typical fMRI blood oxygen level-dependent contrasts, summarized as contrast-to-noise improvements. Detailed theoretic predictions are difficult, however, because several factors influence possible clinical benefits (eg, increased artifacts level, scaling of physiologic noise with field strength, dependence of effects on the voxel size). Therefore, experimental investigations on how much functional signals in- 




FIG 1. Improved functional signal strength in a patient with tumor generated by a 7T (Siemens Magnetom) ultra-high-field system within the Wernicke language area (overt speech design, Study Group Clinical $f M R I^{4}$ ).

crease with field strength are urgently needed. A first study comparing $3 \mathrm{~T}$ and $7 \mathrm{~T}$ motor cortex signals in patients with brain tumors provided promising results: Five of 6 functional parameters indicated improved sensitivity for $7 \mathrm{~T}$, even at a standard resolution $(1.8 \times 1.8 \times 3 \mathrm{~mm}$ voxel size $)$ and with standard clinical procedures. ${ }^{4}$ Corresponding language data (work in progress) achieved with an overt response paradigm that includes various subcomponents of language processing ${ }^{5}$ indicate a similar 7T benefit, provided that the artifacts situation can be controlled (Fig 1).

Although the current reliability of fMRI is adequate for most clinical demands, there is still the potential for further improvement. ${ }^{6}$ Given that a number of high-quality paradigms have been published by several expert clinical groups for the most important tasks in the interim, significant further progress is most likely to be achieved by new techniques that improve functional signal strength, such as clinical 7T systems. Research into this issue is of the utmost importance. If initial results can be confirmed, there is a good chance that such studies will fuel a hardware revolution similar to the general shift of clinical work from 1.5T to 3T during the past 10 years.

\section{ACKNOWLEDGMENTS}

I am grateful to M. Wurnig, F. Fischmeister, E. Matt, E. Auff, A. Geißler (Study Group Clinical fMRI, Department of Neurology, High Field MR Center, Medical University of Vienna, Austria), and S. Trattnig and S. Robinson (Department of Radiology, High Field MR Center, Medical University of Vienna, Austria) for contributions to the clinical fMRI data. Generation of the data was supported by the Vienna Spots of Excellence Program of the Center of Innovation and Technology, City of Vienna (ZIT), Austria, and the Austrian Science Fund (FWF) grants KLI264 and P23611.

\section{REFERENCES}

1. O'Shea J, Boudrias MH, Stagg CJ, et al. Predicting behavioural response to TDCS in chronic motor stroke. Neuroimage 2013; pii:S1053-8119(13)00598-603

2. Szaflarski JP, Allendorfer JB, Banks C, et al. Recovered vs. not-recovered from post-stroke aphasia: the contributions from the dominant and non-dominant hemispheres. Restor Neurol Neurosci 2013;31:347-60

3. Zacà D, Jarso S, Pillai JJ. Role of semantic paradigms for optimization of language mapping in clinical fMRI studies. AJNR Am J Neuroradiol 2013;34:1963-68

4. Beisteiner R, Robinson S, Wurnig M, et al. Clinical fMRI: evidence for a $7 \mathrm{~T}$ benefit over $3 \mathrm{~T}$. Neuroimage 2011;57:1015-21

5. Gartus A, Foki T, Geissler A, et al. Improvement of clinical language localization with an overt semantic and syntactic language functional MR imaging paradigm. AJNR Am J Neuroradiol 2009; 30:1977-85

6. Wurnig MC, Rath J, Klinger N, et al. Variability of clinical functional MR imaging results: a multicenter study. Radiology 2013;268: $521-31$

R. Beisteiner Study Group Clinical fMRI Department of Neurology High Field MR Center Medical University of Vienna Vienna, Austria 